\title{
Effectiveness of etofenamate for treatment of knee osteoarthritis: a randomized controlled trial
}

\author{
This article was published in the following Dove Press journal: \\ Therapeutics and Clinical Risk Management \\ 14 November 2016 \\ Number of times this article has been viewed
}

\author{
Savaș Güner' \\ Mehmet Ata Gökalp' \\ Abdurrahim Gözen' \\ Seyyid Șerif Ünsal' \\ Șükriye İlkay Güner ${ }^{2}$ \\ 'Department of Trauma and \\ Orthopedic Surgery, Medical School, \\ Yuzuncu Yil University, ${ }^{2}$ School \\ of Health, Yuzuncu Yil University, \\ Van, Turkey
}

\begin{abstract}
The intramuscular application of etofenamate in the treatment of knee osteoarthritis was not observed in the existing English language literature. The objectives of this study were to compare the efficacy of etofenamate versus hyaluronic acid (HA) in reducing joint pain and functional improvement for mild to moderate knee osteoarthritis. The patients were randomly divided into etofenamate $(n=29)$ and HA $(n=30)$ groups. Intramuscular etofenamate injection was administered as a series of seven intramuscular injections at intervals of 1 day. Intra-articular HA injection was administered as a series of three intra-articular injections at intervals of 1 week. Clinical evaluation was made before the first injection and again both 6 and 12 months after the last injection. The evaluation consisted of patient-assessed pain on a visual analog scale (VAS) and on the Western Ontario and McMaster Universities Osteoarthritis Index (WOMAC). Statistical significance was found for the etofenamate group when comparing preinjection with 12 months postinjection VAS scores $(P<0.05)$. Statistical significance was also found for the HA group when comparing preinjection with 12 months postinjection VAS and WOMAC scores $(P<0.05)$. However, there was no significant difference between the etofenamate and HA groups in terms of VAS or WOMAC scores measured at 12 months after injection $(P>0.05)$. Results from this study indicated that, etofenamate treatment was not significantly more effective than HA treatment. However, both methods were effective and successful in treating knee osteoarthritis.
\end{abstract}

Keywords: knee osteoarthritis, arthralgia, treatment, etofenamate, nonsteroidal anti-inflammatory drugs

\section{Introduction}

Knee osteoarthritis (OA) is a frequent and progressive degenerative joint disease that occurs primarily in older adults. Though the pathogenesis is not fully clear, it is characterized by hypertrophy of the bone at the margins, erosion of the articular cartilage, and subchondral sclerosis. ${ }^{1}$

There is no present treatment to prevent disease progression. Goals for managing $\mathrm{OA}$ are to decrease pain, improve and maintain sufficient joint range of motion, and limit functional impairment. Treatment is individualized and often involves a combination of nonpharmacological (eg, weight loss, lifestyle adaptations, exercise, and physical therapy) and pharmacological approaches (eg, paracetamol, nonsteroidal antiinflammatory drugs [NSAIDs], and visco-supplementation). Patients with OA generally use oral and topical NSAIDs to decrease pain. ${ }^{2,3}$ It is suggested that NSAIDs should be administered to patients who do not obtain adequate pain relief with paracetamol. ${ }^{2,4}$ Intra-articular hyaluronic acid (HA) treatments for knee OA are often performed as an option when oral medication is unsuccessful. Surgery is usually reserved for patients when all other methods have failed to alleviate pain and disability. ${ }^{3}$ 
Etofenamate is an NSAID. Etofenamate is used for the treatment of joint and muscular pain. Etofenamate acts by inhibiting cyclo-oxygenase, an enzyme involved in prostaglandin synthesis possess analgesic and anti-inflammatory properties. ${ }^{3}$ Intramuscular application of etofenamate in treatment of knee OA was not observed in the existing English language literature. The objectives of this study were to compare the efficacy of etofenamate versus HA in reducing joint pain in mild to moderate knee OA.

\section{Materials and methods Study design}

The study was conducted between June 2011 and June 2012. This prospective randomized controlled and open study was carried out with the approval of the ethics committee of Yuzuncu Yil University (registration number 07062012.09) and adhered to the Declaration of Helsinki. Written informed consent was obtained from each patient participating in the study. The patients were randomly allocated to the etofenamate group or the HA group. Randomization was performed by computer generated random allocations sequence by simple randomization. The randomization sequence was concealed from investigators until interventions were assigned. The allocation ratio was $1: 1$.

The basic characteristics of both groups, including height, weight, and body mass index, were recorded. The patients were radiologically examined, and blood samples were collected for hemogram and biochemical analysis. Standard weight-bearing anteroposterior and lateral radiography of the knees in full extension was performed, and each film was classified by a single experienced and blinded observer using the Kellgren-Lawrence grading scale ${ }^{5}$ (grade 0: no radiographic features of $\mathrm{OA}$ are present; grade 1: doubtful joint space narrowing [JSN] and possible osteophytic lipping; grade 2: definite osteophytes and possible JSN on anteroposterior weight-bearing radiograph; grade 3: multiple osteophytes, definite JSN, sclerosis, possible bony deformity; grade 4: large osteophytes, marked JSN, severe sclerosis, and definite bony deformity).

Data of the study were obtained at orthopedic clinic of Yuzuncu Yil University. Inclusion criteria were those aged 50-70 years, standard radiographic criteria for symptomatic mild or moderate knee OA (Kellgren-Lawrence 2 and 3), and pain with the regular use of NSAIDs or other analgesics. In the study, exclusion criteria were biochemical analysis abnormality, active peptic ulcer, pregnancy, secondary arthritis, hypertension, previous knee surgery, sensitivity to HA or other NSAIDs, a history of chronic infection, such as hepatitis, other systemic diseases, such as severe cardiac, renal, or hepatic diseases, a history of allergies, asthma, cardiac or renal failure, or a history of drug or alcohol abuse.

Intra-articular injection of HA was performed under sterile conditions by the same physician in all patients. Intramuscular injection of etofenamate was performed under sterile conditions by the same nurse (SIG).

\section{Groups}

The patients were randomly divided into two groups:

1. Etofenamate group ( $n=29)$ : Intramuscular etofenamate (Flexo $^{\circledR}$ ampul, $100 \mathrm{mg} / 2 \mathrm{~mL}$ Santa Farma, Istanbul, Turkey) injection was administered as a series of seven intramuscular injections at intervals of 1 day (Figure 1). Intramuscular injections were made in the supero-lateral quadrant of the gluteal region. In addition, a proton pump inhibitor (lansoprazole $30 \mathrm{mg}$ /day) was given for 2 weeks for patients with gastrointestinal system problems.

2. HA group $(n=30)$ : Intra-articular HA (Orthovisc ${ }^{\circledR}$, $30 \mathrm{mg} / 2 \mathrm{~mL}$, Biomeks, Istanbul, Turkey) injection was administered as a series of three intra-articular injections at intervals of 1 week (Figure 1). Knee injections were made via a supero-lateral approach. Layer-by-layer local anesthesia was performed using lidocaine 1\%. Arthrocentesis was carefully performed prior to each injection to remove any effusion. When the needle was in the correct position, the injection was performed. After resting for 1 hour, the patient was allowed to walk and to return home. The patient was recommended to rest at home until the next day.

\section{Follow-up}

The follow-up period was 12 months. In all, the patients were evaluated before the treatment and 6 and 12 months after the last injection. Any treatment for both groups was not administered except the recommended treatment.

\section{Clinical evaluation}

Patients were evaluated blinded to the group type. The evaluation consisted of patient-assessed pain on a visual analog scale (VAS) and The Western Ontario and McMaster Universities (WOMAC) OA Index. ${ }^{6}$ Clinical evaluation was made by a nurse (SIG). The VAS is a $10 \mathrm{~cm}$ line beginning at 0 and ending at 10 . The score is marked at the point on the line that corresponds with the patient's pain level. On this scale, 0 reflects a total absence of symptoms and 10 indicates the worst imaginable pain or stiffness. The WOMAC 


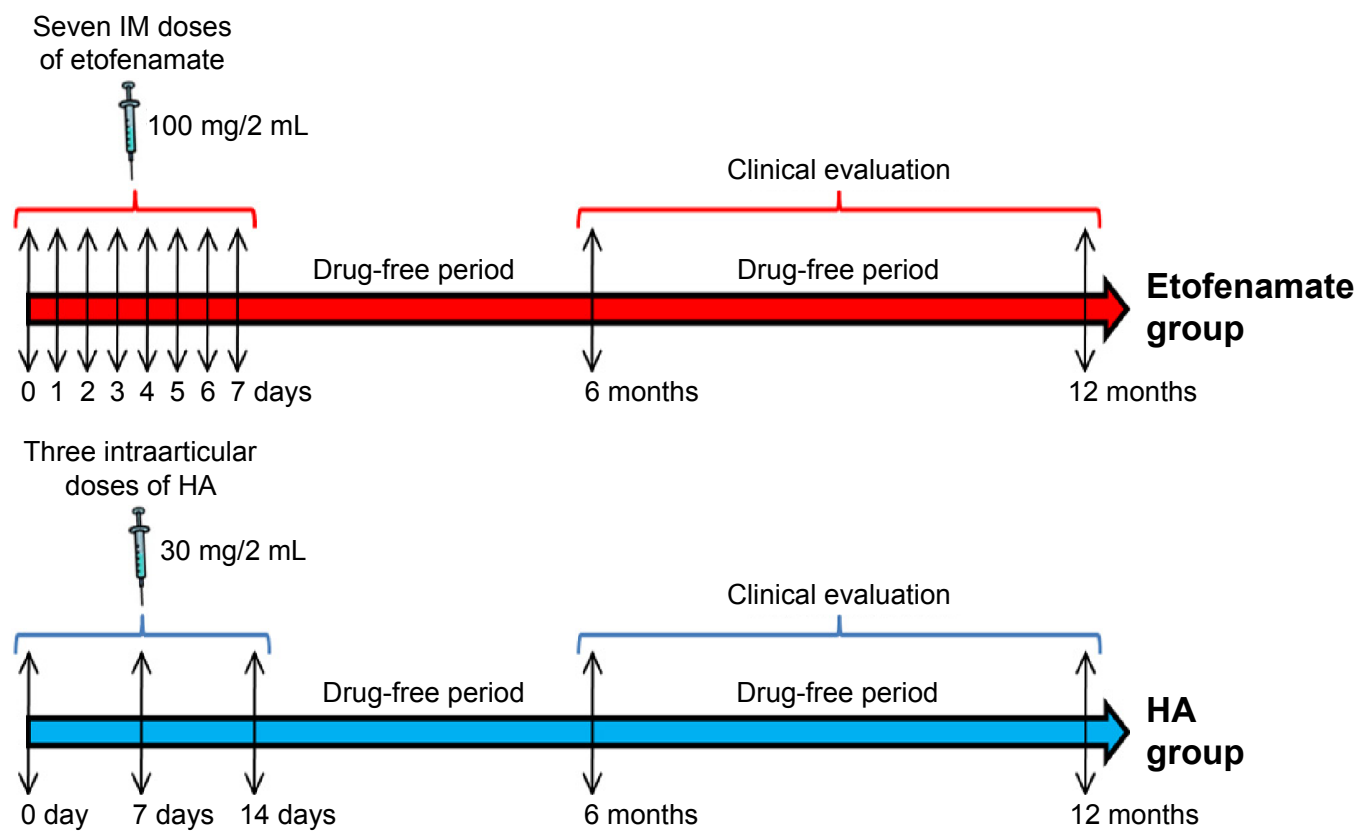

Figure I Protocols of administration.

Note: Clinical evaluation (VAS and WOMAC OA index) was made before the first injection and 6 and 12 months after the last injection.

Abbreviations: VAS, visual analog scale; WOMAC, Western Ontario and McMaster Universities Osteoarthritis Index; OA, osteoarthritis; IM, intramuscular; HA, hyaluronic acid.

OA index is used for clinical trials in patients with knee OA. The WOMAC OA index is a disease-specific selfadministered health status measure that is widely accepted as reflective of OA disease activity. The original index consists of 24 questions (five on pain, two on stiffness, and 17 on physical function). Individual question responses are assigned a score of between 0 (none) to 4 (extreme) and then added together to form a score ranging from 0 (best) to 96 (worst). There are three sections to the WOMAC score. Section A addresses the amount of pain (five questions), section $\mathrm{B}$ addresses the amount of joint stiffness (two questions), and section $\mathrm{C}$ addresses aspects of physical functioning (17 questions). ${ }^{6}$

\section{Statistical analysis}

Formula for sample size was used for sample size calculation.? Descriptive statistics for studied variables (characteristics) were presented as the mean, standard deviation (SD), and minimum and maximum values. Repeated measurement analysis of variance with two factors (months are repeated factors) was performed to compare the mean of group and month for the WOMAC and VAS scores. One-way analysis of variance was also performed for age, height, weight, and body mass index. Following analyses of variance, the Tukey multiple comparison test was carried out to determine different group mean. Statistical significance levels were considered to be 5\%, and SPSS version 13 (SPSS Inc., Chicago, IL, USA) statistical program was used for all statistical computations.

\section{Results}

According to sample size calculation by simple random sampling method, sample size was found as 26 for each group (confidence level 95\%). A total of 70 patients were eligible for the study. Before randomization, eight patients were excluded from the study (five patients were excluded from the study because of a history of a peptic ulcus, one patient was excluded because of cardiac failure, and two patients were excluded because of hypertension). None of patients chose to withdraw. Three patients (two patients in etofenamate group and one patient in HA group) who did not come to follow-ups regularly were omitted from the study. In all, 59 patients who were followed up regularly completed the study. Drop-out rate was $4.8 \%$. The flowchart is shown in Figure 2.

Baseline demographic and clinical characteristics between the etofenamate and HA groups are shown in Table 1. Of the total knees studied, we examined 28 (48.3\%) left knees and 30 (51.7\%) right knees. Height, weight, and body mass index were examined, and there were no statistically significant differences between groups $(P>0.05)$.

The follow-up duration for the etofenamate group was 12 months. Using the VAS and WOMAC scores, the mean 


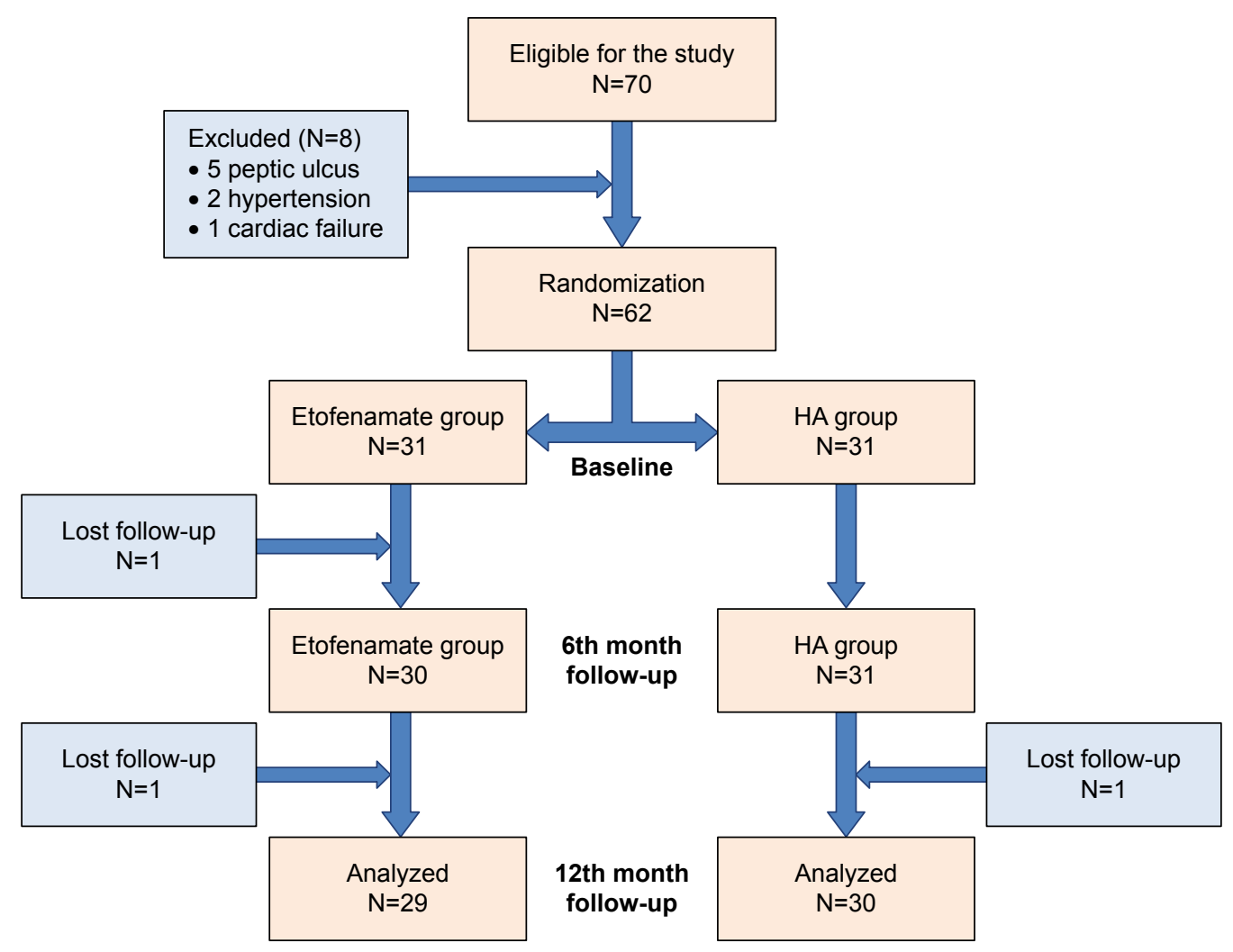

Figure 2 Flow chart of the patients in the study.

Abbreviation: $\mathrm{HA}$, hyaluronic acid.

preinjection level was 8.67 ( $\mathrm{SD} \pm 1.14$, range 6-10) and 82.89 (SD \pm 8.79 , range 61-96), respectively. Postinjection 6 months using the VAS and WOMAC scores demonstrated that pain decreased to a mean level of 1.93 ( $\mathrm{SD} \pm 1.64$, range $0-5$ ) and 15.07 ( $\mathrm{SD} \pm 15.45$, range $1-56$ ), respectively. Postinjection 12 months using the VAS and WOMAC scores demonstrated that pain decreased to a mean level of 2.30 ( $\mathrm{SD} \pm 2.33$, range $0-7$ ) and 18.59 ( $\mathrm{SD} \pm 16.71$, range $2-68$ ),

Table I Baseline demographic and clinical characteristics

\begin{tabular}{llll}
\hline Characteristic & Etofenamate group & HA group & P-value \\
\hline Sex $(\mathbf{n})$ & & & $>0.05$ \\
Male & 5 & 3 & \\
Female & 24 & 27 & $>0.05$ \\
Age (years) & & & \\
Minimum & 50 & 50 & \\
Maximum & 70 & 70 & \\
Mean & 61.3 & 62.5 & \\
BMI (kg/m $\left.{ }^{2}\right)$ & & & \\
Minimum & 22.72 & 21.50 & \\
Maximum & 35.65 & 35.11 & \\
Mean & 28.73 & 27.54 & \\
K/L grading & & & \\
Grade 2 & 17 & 15 & \\
Grade 3 & 12 & 15 & \\
\hline Abbriation &
\end{tabular}

Abbreviations: BMI, body mass index; HA, hyaluronic acid; K/L, Kellgren-Lawrence. respectively. The follow-up duration was also 12 months for the HA group. Using the VAS and WOMAC scores, the mean preinjection pain levels were 9.15 ( $\mathrm{SD} \pm 1.06$, range 7-10) and 86.89 ( $\mathrm{SD} \pm 4.84$, range 74-95), respectively. The mean postinjection VAS and WOMAC scores at 6 months were 1.74 ( $\mathrm{SD} \pm 1.89$ range $0-8$ ) and 15.71 ( $\mathrm{SD} \pm 21.65$, range 2-74), respectively. The mean postinjection VAS and WOMAC scores at 12 months were 2.26 ( $\mathrm{SD} \pm 2.56$ range 0-9) and 18.07 ( $\mathrm{SD} \pm 22.39$, range 2-78), respectively. The mean preinjection and 6- and 12-month follow-up VAS and WOMAC scores are presented in Figures 3 and 4.

Statistical significance was found for the etofenamate group when comparing the preinjection and 6 months postinjection VAS and WOMAC scores $(P<0.05)$ and preinjection and 12 months postinjection VAS and WOMAC scores $(P<0.05)$. Statistical significance was also found for HA group when comparing the preinjection and 6 months postinjection VAS and WOMAC scores $(P<0.05)$ and preinjection and 12 months postinjection VAS and WOMAC scores. However, there was no significant difference between the etofenamate and HA groups in terms of VAS or WOMAC scores measured at 12 months after injection $(P>0.05)$. There was no 


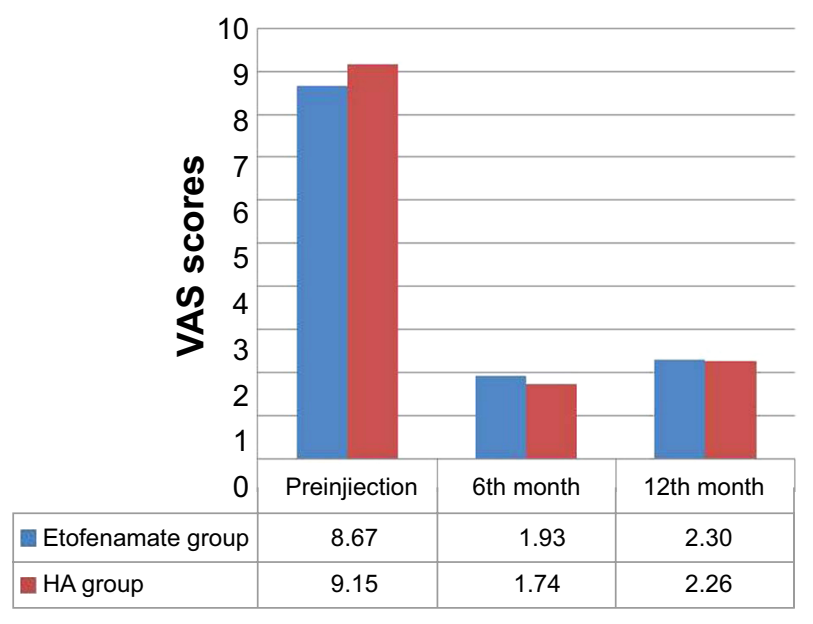

Figure 3 The mean preinjection and 6- and 12-month follow-up VAS scores. Abbreviations: HA, hyaluronic acid; VAS, visual analog scale.

significant difference between the etofenamate and HA groups in terms of VAS or WOMAC scores measured between 6 and 12 months.

No side effects or complications occurred in patients with intramuscular etofenamate therapy or intra-articular HA therapy.

\section{Discussion}

Etofenamate is an NSAID of the flufenamic acid derivative group and has strong analgesic and anti-inflammatory activity. Etofenamate has poor oral bioavailability. Therefore, it is only administered via topical and intramuscular methods in clinical practice. The effectiveness of intramuscular etofenamate in numerous painful situations has been confirmed in a number of studies. ${ }^{8-10}$ The plasma concentrations of etofenamate increase less rapidly because etofenamate

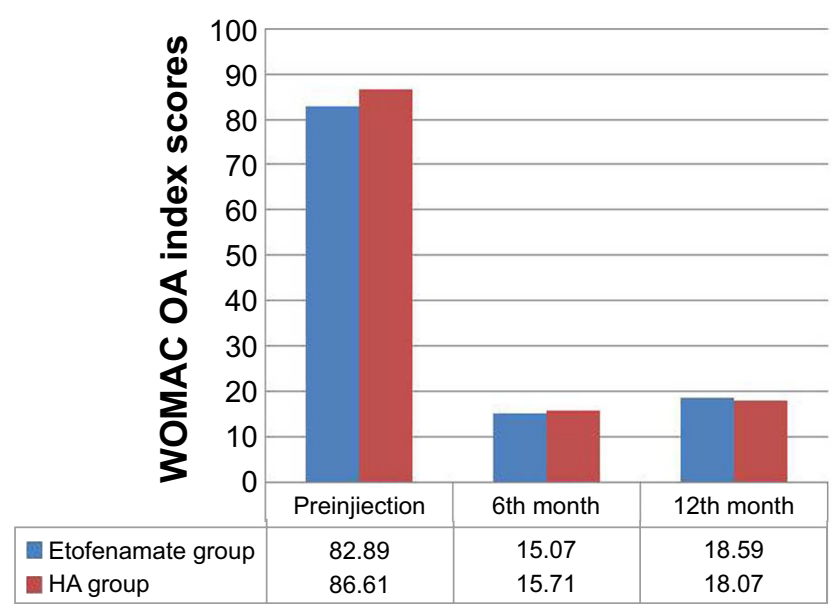

Figure 4 The mean preinjection and 6- and I2-month follow-up WOMAC scores. Abbreviations: HA, hyaluronic acid; OA, osteoarthritis; WOMAC, Western Ontario and McMaster Universities Osteoarthritis Index. is an oily solution. Relatively high plasma levels persist for up to 10 hours, likely because of a "depot-like" phenomenon resulting from the oily nature of the solution. ${ }^{11}$ Theoretically, this profile would cause a more prolonged action etofenamate.

Numerous types of treatment have a role in the management of the pain of OA. ${ }^{2-4}$ Conservative and surgical treatments both aim at preserving quality of life by decreasing symptoms and facilitating better knee functioning in patients with knee OA. The conservative treatments are usually the first choice, especially in the phase of the disease recognized as late OA or in the presence of associated abnormalities requiring surgery. ${ }^{3}$ Paracetamol is an initial option for reduce pain in knee OA. Because paracetamol has fewer side effects, most physicians suggest using this drug first and then shifting to an NSAID if paracetamol does not provide adequate pain relief. ${ }^{12}$ Oral and topical NSAIDs are frequently used in patients with mild or moderate pain due to knee OA. They are successful drugs in relieving pain and inflammation associated with knee OA. ${ }^{13,14}$ If an oral NSAID is prescribed for a patient with risk factors related to the gastrointestinal system, the use of a combined nonselective NSAID with a gastro-protective agent should be considered. ${ }^{15}$ We also gave a proton pump inhibitor for patients with etofenamate treatment. In our study, we did not observe gastrointestinal complications associated with using etofenamate. Oral NSAIDs should be used only for the shortest possible period of time. However, many patients with OA have to use NSAIDs for prolonged periods in order to provide permanent pain reduction. The long-term use of NSAIDs does not delay the progression of OA and can increase the risk of side effects in patients. ${ }^{4} 15$ Although we used etofenamate for a short period, we obtained an analgesic effect for a duration of 12 months.

HA is a natural substance in the synovial fluid in joints. It plays the roles of a lubricant and a shock absorber in a healthy joint. OA reduces the synovial fluid's ability to lubricate and protect the joint. Although the precise mechanism of the effects of HA treatment is not obvious, HA is believed to increase the viscoelasticity of the synovial fluid and restore the trans-synovial flow. ${ }^{16,17}$ Intra-articular HA injections are frequently used in the treatment of knee OA. The valuable effects of HA and its derivates in the treatment of knee OA were reported in the trial. ${ }^{16-18}$ There are case reports of observed local and systemic side effects associated with intra-articular HA injection in the literature. ${ }^{19,20}$ However, we did not observe any adverse effects associated with intra-articular HA injections in our study. 
This study was primarily designed to evaluate the effectiveness of treatment of knee OA using etofenamate. A comparison was made between etofenamate and HA therapy in knee OA. No significant differences were found in the VAS pain scores and WOMAC OA indexes at the 6 th and 12th months between etofenamate and HA groups $(P>0.05)$. A surgical treatment option should be considered a last resort. Total knee replacement (TKR) operation is a frequently performed surgical option in patients with knee OA. The major indication of TKR is relief of pain associated with knee OA if nonsurgical treatment has not been efficient. ${ }^{21}$ In numerous cases, TKR operation in younger patients should perhaps be delayed because of the risk of revisions, pain from overuse, or loosening from the bone. The procedure should be delayed or avoided totally in older patients or patients with comorbidities that may increase surgery complication risks. Furthermore, delaying a TKR operation may reduce the need for revision surgery in the future. Revision operations are usually more costly than primary procedures. They are more difficult to perform and have poor results in comparison with primary TKR. ${ }^{22}$ Clinical trials have showed that intraarticular HA can delay a need for TKR due to a pain decrease in most patients. ${ }^{23,24}$ According to the results obtained in this study, we believe that there are also reasons to delay TKR with etofenamate treatment. Thus, we believe that the need of TKR can be further delayed by the application of etofenamate treatment between NSAID and HA treatments.

People who are above age 65 are influenced by knee OA in a widespread manner because of continuing decrease in birth rates. In relation to this, total increase in life expectancy causes a worrisome tendency in the prevalence of knee OA for the coming older population. ${ }^{25}$ It was observed that the average age of both two groups was lower in comparison to the literature. ${ }^{26,27} \mathrm{We}$ think that the reason for this situation stems from people working in demanding agricultural activities (especially in the female population). The most important limitation of the present study is the relatively short follow-up period in the study group. Long-term follow-up results are needed to confirm further studies. Other limitations of our study were not being blinded to the patients, absence of intention to treat analysis, and absence of a placebo group for assessing the treatment's success. Further studies should include a placebo group.

\section{Conclusion}

From the current study, etofenamate treatment was not significantly more effective than HA treatment. Both methods resulted in similar results in treating knee OA.

\section{Disclosure}

The authors report no conflicts of interest in this work.

\section{References}

1. Di Cesare P, Abramson S, Samuels J. Pathogenesis of osteoarthritis. In: Firestein GS, Kelley WN, editors. Kelley's Textbook of Rheumatology. Philadelphia: Saunders Elsevier; 2009:1525-1540.

2. Zhang W, Moskowitz RW, Nuki G, et al. OARSI recommendations for the management of hip and knee osteoarthritis, Part II: OARSI evidencebased, expert consensus guidelines. Osteoarthritis Cartilage. 2008; 16(12):137-162.

3. Grainger R, Cicuttini FM. Medical management of osteoarthritis of the knee and hip joints. Med J Aust. 2004;180(5):232-236.

4. Zhang W, Nuki G, Moskowitz RW, et al. OARSI recommendations for the management of hip and knee osteoarthritis: part III: changes in evidence following systematic cumulative update of research published. Osteoarthritis Cartilage. 2010;18(4):476-499.

5. Kellgren JH, Lawrence JS. Radiological assessment of osteo-arthrosis. Ann Rheum Dis. 1957;16(4):494-502.

6. Bellamy N, Buchanan WW, Goldsmith CH, Campbell J, Stitt LW. Validation study of WOMAC: a health status instrument for measuring clinically important patient relevant outcomes to anti-rheumatic drug therapy in patients with osteoarthritis of the hip or knee. J Rheumatol. 1988;15(12):1833-1840.

7. Barttlet JE, Kotrlik JW, Higgins CC. Organizational research: determining appropriate sample size in survey research. Inf Technol Learn Perform J. 2001;19(1):43-50.

8. Hensler G. Behandlung frischer stumpfer traumata. Ergebnisse einer einfachblind-prüfung mit etofenamate bzw diclofenac-Na injektionslösung [Treatment of recent blunt traumas. Results of a single-blind-test injection solution with etofenamate or diclofenac-Na]. Z Allgemein Med. 1990;66(5):255-258. German.

9. Miehlke K. Injektionsbehandlung des akuten lumbalsyndroms: partment etofenamat und diclofenac im vergleich [Injection treatment of acute lumbar syndrome: partment etofenamate and diclofenac compared]. Therapiewoche. 1990;40(12):462-465. German.

10. Sousa MMJ. Etofenamato no tratamento da cólica renal [Etofenamate in the treatment of renal colic]. Acta Urol Port. 1996;13(1-4):43-46. Portuguese.

11. Beckermann B, Bock E, Kamp R, Dell HD. Plasma level studies on healthy volunteers after intramuscular injection of various doses of etofenamate in an oily solution. Arzneimittel-Forschung. 1990;40(3): 305-311.

12. Hochberg MC, Altman RD, Brandt KD, et al. Guidelines for the medical management of osteoarthritis. Part II. Osteoarthritis of the knee. American college of rheumatology. Arthritis Rheum. 1995;38(11): $1541-1546$.

13. McKenna F, Borenstein D, Wendt H, Wallemark C, Lefkowith JB, Geis GS. Celecoxib versus diclofenac in the management of osteoarthritis of the knee. Scand J Rheumatol. 2001;30(1):11-18.

14. Zhao SZ, McMillen JI, Markenson JA, et al. Evaluation of the functional status aspects of health-related quality of life of patients with osteoarthritis treated with celecoxib. Pharmacotherapy. 1999;19(11): 1269-1278.

15. Hochberg MC, Altman RD, Toupin AK, et al. American college of rheumatology 2012 recommendations for the use of nonpharmacologic and pharmacologic therapies in osteoarthritis of the hand, hip, and knee. Arthritis Care Res (Hoboken). 2012;64(4):465-474.

16. Ghosh P, Holbert C, Read R, Armstrong S. Hyaluronic acid (hyaluronan) in experimental osteoarthritis. J Rheumatol Suppl. 1995;43(2): $155-157$.

17. Gotoh S, Onaya J, Abe M, et al. Effects of the molecular weight of hyaluronic acid and its action mechanism on experimental joint pain in rats. Ann Rheum Dis. 1993;52(11):817-822.

18. Goldberg RL, Toole BP. Hyaluronate inhibition of cell proliferation. Arthritis Rheum. 1987;30(7):769-778. 
19. Martens PB. Bilateral symmetric inflammatory reaction to hylan G-F 20 injection. Arthritis Rheum. 2001;44(4):978-979.

20. Allen E, Krohn K. Adverse reaction to Hylan GF-20. J Rheumatol. 2000;27(6):1572.

21. Kane RL, Saleh KJ, Wilt TJ, et al. Total knee replacement. Evidence Report/Technology Assessment no. 86. Rockville, MD: Agency for Healthcare Research and Quality; 2003:1-8.

22. Burns AW, Boume RB, Cheswonh BM, MacDonald SJ, Rorabeck CH. Cost effectiveness of revision total knee arthroplasty. Clin Orthop Relat Res. 2006;446(5):29-33.

23. Waddell DD. Bricker DC. Total knee replacement delayed with hylan G-F 20 use in patients with grade IV osteoarthritis. J Manag Care Pharm. 2007;13(2):113-121.
24. Waddell D, Rein A, Panarites C, Coleman PM, Weiss C. Cost implications of introducing an alternative treatment for patients with osteoarthrilis of the knee in a managed care setting. Am J Manag Care. 2001; 7(10):981-991.

25. Ringdahl E, Pandit S. Treatment of knee osteoarthritis. Am Fam Physician. 2011;83:1287-1292.

26. Felson DT, Naimark A, Anderson J, Kazis L, Castelli W, Meenan RF. The prevalence of knee osteoarthritis in the elderly. The framingham osteoarthritis study. Artritis Rheum. 1987;30(8):914-918.

27. Bhatia D, Bejarano T, Novo M. Current interventions in the management of knee osteoarthritis. J Pharm Bioallied Sci. 2013;5(1):30-38.

\section{Publish your work in this journal}

Therapeutics and Clinical Risk Management is an international, peerreviewed journal of clinical therapeutics and risk management, focusing on concise rapid reporting of clinical studies in all therapeutic areas, outcomes, safety, and programs for the effective, safe, and sustained use of medicines. This journal is indexed on PubMed Central, CAS,
EMBase, Scopus and the Elsevier Bibliographic databases. The manuscript management system is completely online and includes a very quick and fair peer-review system, which is all easy to use. Visit http://www.dovepress.com/testimonials.php to read real quotes from published authors.

Submit your manuscript here: http://www.dovepress.com/therapeutics-and-clinical-risk-management-journal 